\title{
Integrase Strand Transfer Inhibitors \& Weight Gain in Children with Perinatal HIV
}

Gianna Fernandez', Alka Khaitan²

${ }^{1}$ Indiana University School of Medicine; ${ }^{2}$ Department of Pediatric Infectious Diseases, Riley Hospital for Children at Indiana University Health

Background: In the past, HIV infection was associated with weight loss due to metabolic wasting. Now with early initiation of antiretroviral therapy (ART) an increasing number of patients are being classified as overweight/ obese both prior to initiation and during the course of ART. Certain integrase strand transfer inhibitors (INSTIs) have been associated with weight gain in the adult population, but data in the pediatric population is lacking. This study explores the association of ART with an INSTI backbone and changes in weight and body mass index (BMI) in children with perinatal HIV who are followed in the HIV clinic at Riley Hospital.

Methods: We performed a retrospective review of 59 patients with perinatally acquired HIV from 2016 to 2021. Weight, BMI, z-score, blood pressure (BP) percentiles, and lipid panels were recorded from the patients' most recent visit. BMI and BP percentiles were compared between patients on Bictegravir, Dolutegravir, and Elvitegravir. For patients currently on an INSTIbackbone ART regimen, BMI was compared before and up to 5 years after INSTI initiation.

Results: In our cross-sectional analysis between patients on Bictegravir, Dolutegravir, and Elvitegravir there was no significant difference in BMI $(p=0.859)$, systolic BP percentile $(p=0.188)$, or diastolic BP percentile $(p=0.541)$. In our longitudinal analysis, we observed a significant increase in BMI over 3 years compared to pre-INSTI initiation ( $\mathrm{p}=0.049)$. In addition, we observed an upwards trend with patients on Bictegravir $(\mathrm{p}=0.094)$ and Elvitegravir $(\mathrm{p}=0.121)$.

Conclusion/Impact: Our study suggests that ART regimens with an INSTI backbone are associated with greater increases in BMls. Our study is limited by a small n, but provides pilot data to direct future studies. It also illustrates that emphasis on healthy body weight maintenance may be necessary when patients are placed on ART regimens with INSTI backbones. 